\title{
El consumo político en Argentina: estrategias desarrolladas por organizaciones sin fines de lucro
}

\author{
Marcos Rodríguez de Ancos
}

\section{RESUMEN}

El consumo político (también llamado consumo responsable, consumo ético o activismo del consumidor) viene configurándose como un factor clave para la sostenibilidad global y el impulso de la responsabilidad social corporativa. El estudio de este fenómeno se ha centrado en los países más desarrollados y el conocimiento de sus características en los países en vías de desarrollo ha recibido una atención mucho menor. Este trabajo analiza la situación del consumo político en Argentina a través de las estrategias desarrolladas por organizaciones sin fines de lucro para su fomento. Aplicando la metodología del estudio de casos, se examinan las iniciativas más relevantes y se clasifican siguiendo diferentes criterios teóricos. Los resultados indican un predominio de estrategias de cambio basadas en relaciones de colaboración y del tipo bottom-up (de abajo a arriba).

PALABRAS CLAVE: Consumo político, Argentina, organizaciones sin fines de lucro, estrategias para el cambio, consumo responsable, responsabilidad social corporativa.

CLAVES ECONLIT: E29, D79, L31, M14.

Cómo citar este artículo / How to cite this article: RODRÍGUEZ DE ANCOS, M. (2016): "El consumo político en Argentina: estrategias desarrolladas por organizaciones sin fines de lucro", CIRIEC-España, Revista de Economía Pública, Social y Cooperativa, 86, 251-279.

Correspondencia: Marcos Rodríguez de Ancos, Máster en Sostenibilidad y Responsabilidad Social Corporativa, Universidad Nacional de Educación a Distancia (UNED) y Universidad Jaume I (UJI). E-mail: marcos.rod.da@gmail.com. 


\section{EXPANDED ABSTRACT}

\section{Political consumption in Argentina: strategies developed by non-profit organizations}

In the current context of depletion of natural resources and global warming, the model of consumption of opulent Northern countries - replicated by many Southern countries - has become one of the causes of the environmental crisis and a source of social conflict. This is why it is especially interesting to reflect on alternative forms of consumption that stem from a critical analysis of the prevailing model and seek to generate social reform based on daily actions.

Political consumption, understood as a new social movement, has so far had a modest theoretical trajectory and limited - although not negligible - impact in practical terms. In addition, in Argentina, the country that is the subject of this research, political consumption has barely been studied to date. It is hoped that this research project will add both academic and civic value to a little-explored area.

The research focuses on analysing the role of Argentina's not-for-profit organisations (NPOs) in promoting this phenomenon.

On the theoretical side, we approximate institutional theory and the relationship with corporate social responsibility (CSR) to the NPOs' capacity to influence the generation of changes in company and consumer attitudes, that is to say, to have an impact on the supply and demand for goods and services. Therefore, NPOs are analysed as civil society articulators to generate changes that point to a model of sustainable consumption.

Along these lines, following Bendell (2007), NPOs can develop different types of strategies to influence corporations, depending on whether they develop collaborative or confrontational relations and whether these are within or outside the market.

The practical side is the repertory of actions the political consumer can undertake, either individually or collectively. Again, we determine the typology using two core ideas: whether the actions are purchasing or not purchasing and whether they occur by means of conventional or alternative channels.

After the theoretical framework, we present an analysis of the political consumption context in Argentina, firstly through the evolution of the not-for-profit sector from colonial times up to the present; secondly by means of the model proposed by Thogersen (2010), which includes market as well as poli- 


\section{EL CONSUMO POLÍTICO EN ARGENTINA: ESTRATEGIAS DESARROLLADAS POR ORGANIZACIONES SIN FINES DE LUCRO}

tical factors; and thirdly, taking into account the contemporary characteristics of the not-for-profit sector. From this analysis we deduce that even though the Argentinian context cannot be called adverse, it is far from presenting the conditions of those countries where the movement is more developed.

The method used in this research is case studies. Specifically, using theoretical criteria, six organisations were selected according to their area of activity, size and geographical scope. All had to comply with two criteria: defence of a sustainable development model as a central axis of their activity and the presence of political consumption or one of its types of action as a line of work or objective.

In order to get to know the initiatives put into practice by different NPOs, firstly a process of investigation and detailed analysis of webpages, annual reports and profiles on social networks was undertaken; secondly, a telephone interview of a leading person in each organisation; and finally, an analysis of their presence in the media.

As far as the results are concerned, we observe that, in relation to the assessment by the NPOs in the Argentinian context of political consumption, all the people interviewed agree that it is a very underdeveloped movement in this country, pointing particularly to the scarcity of demand.

We have divided the global analysis of the main initiatives developed by the NPOs into three parts.

The first relates to the strategies of change used to foment political consumption. Here, going back to the typology of Bendell (2007), we find that strategies based on cooperation are in the overwhelming majority and that the predominant place of activity is outside the market.

The second gathers together the alternatives on which the NPOs have an impact, in relation to the actions the consumer can take. In contrast to the previous part, we observe a more even distribution among the four defined types. Nevertheless, we can highlight the most recurrent: smart shopping in fair trade and organic agriculture and simplification centred on recycling, reusing, energy saving and reducing the level of consumption.

The third part refers to the agent on which the organisations act in their projects and campaigns. We observe an important focus on the impact on citizens, well above that on companies and the public administration.

From the analysis of the results we can extract different conclusions. In their role as political consumption mobilisers, Argentinian NPOs support establishing cooperative relationships with the other party without seeking their own economic profit. Therefore, the initiatives they fight for with corporations have less weight. At the same time, in the range of actions that the organisations studied propose to carry out with political consumers we observe an inclination towards alternative forms, smart shopping and simplifying, which implies promoting a change in the system. 
Taking into account the two previous conclusions, the following result is coherent: a predominance of bottom-up strategies of change which centre on the impact on citizens to the detriment of directing efforts towards the most concentrated centres of power.

We can extract implications from the Argentinian case that can be applied to other scenarios in which political consumption is not very developed and where NPOs focus on aid rather than on transformation: changes will depend to a great extent on the efforts of consumers and small businesses and corporations will not feel strongly pressurised to alter their conduct significantly. In this sense, we can certainly consider that the intervention of governments and a growing awareness on the part of citizens is needed to obtain substantial improvements regarding human rights, which both political consumption and CSR can promote, as well as generating major advances on the path of sustainable development.

KEY WORDS: Political consumption, Argentina, non-profit organizations, change strategies, responsible consumption, corporate social responsibility. 


\section{EL CONSUMO POLÍTICO EN ARGENTINA: ESTRATEGIAS DESARROLLADAS POR ORGANIZACIONES SIN FINES DE LUCRO}

\section{1.- Introducción}

Según el Informe sobre Desarrollo Humano de 2013 (PNUD, 2013), los actuales patrones de producción y consumo de los países del Norte son insostenibles y no deben ser replicados por los países del Sur, ya que ello supondría un empeoramiento de la huella ecológica mundial. De esta manera, se pone de manifiesto la necesidad de actuar sobre el modelo de consumo imperante $y$, por tanto, resulta de gran interés reflexionar sobre el rol de los consumidores en la búsqueda de un desarrollo sostenible, ya que éstos constituyen un pilar central de la economía de mercado y sus decisiones agregadas de compra o no compra (demanda) les otorgan un poder capaz de modificar la conducta de las empresas (oferta).

El consumo político es un modo de presionar para conseguir cambios sociales, tanto individual como colectivamente. Esa presión colectiva puede ser articulada y/o impulsada por las organizaciones que integran la llamada sociedad civil. Es aquí donde entran en juego las organizaciones sin fines de lucro (OSFL) a las que nos referiremos durante el trabajo.

El consumo político es un fenómeno de reciente e incipiente desarrollo teórico (Low y Davenport, 2007) y su estudio se ha venido focalizando en los países más desarrollados. Por tal motivo, este trabajo pretende aportar valor en una doble vía: describiendo un fenómeno del que se sabe poco y aplicando una teoría tanto a una población como a un país poco analizados. La pregunta de investigación que se pretende responder es: ¿Qué estrategias de consumo político desarrollan las OSFL en Argentina? El artículo está basado en una investigación exploratoria con objetivos de estudio descriptivos y un enfoque predominantemente cualitativo, cuyo fin es analizar cómo ocurre un fenómeno en su contexto real.

La estructura seguida es la siguiente: en primer lugar, se expone el marco teórico de referencia sobre consumo político, teoría institucional y contexto argentino. En segundo lugar, la metodología, donde se presenta el diseño del estudio. Después se exponen los resultados obtenidos y son encuadrados en el marco teórico. El artículo concluye con las implicaciones de los resultados y con sugerencias sobre líneas de investigación derivadas. 


\section{2.- Marco teórico}

\subsection{Definición y origen del consumo político}

Existe una tendencia creciente entre los académicos a considerar el consumo y las prácticas del consumidor como una forma de política. Según Micheletti y Follesdal (2007: 168) "el consumo político ocurre cuando los consumidores, conscientemente, canalizan su deseo de modificar prácticas ambientales, políticas o éticas reprobables, ya sean institucionales o de mercado, a través de elecciones entre productores y productos". No obstante, esta definición no recoge los actos deliberados de no consumo llevados a cabo por las personas que rechazan el consumismo en que se basa el sistema capitalista por considerarlo insostenible, personas denominadas voluntary simplifiers (Cherrier, 2007; Papaoikonomou, 2012).

Así pues, podemos definir el consumo político como el conjunto de acciones deliberadas de consumo o no consumo motivadas por la consecución del bien común o de beneficios para un determinado colectivo (Valor, 2011). Este fenómeno recibe indistintamente el nombre de consumo ético, consumo político, consumo responsable o activismo del consumidor (Barnett et al., 2005b; Klintman, 2006; Micheletti, 2002; Stolle y Micheletti, 2005). Como veremos a continuación, sus características permiten considerarlo un movimiento social.

El comportamiento responsable del consumidor es el resultado de la asunción por su parte, en tanto ciudadano, de un ámbito más amplio de su responsabilidad para con la sociedad y el medio ambiente, que influye sobre su identidad como consumidor. Por lo tanto, quedan excluidos de la definición de consumo político los actos de consumo que buscan únicamente el beneficio individual (Barnett et al., 2005a). De este modo, las decisiones de compra o no compra se constituyen como una palanca de cambio a favor de los derechos humanos y la conservación medioambiental. Ante las limitaciones actuales del derecho internacional, centrado en los Estados, el consumo político centra el foco en la responsabilidad que deben asumir las empresas y los propios ciudadanos. Por ello, tanto las personas como los colectivos en sus roles de consumidores y productores toman una posición de mayor relevancia como actores para el desarrollo de la sociedad global (Micheletti y Follesdal, 2007). Así pues, el consumo político se configura como un movimiento de defensa de intereses comunes a nivel internacional.

En este sentido, podemos encuadrar este fenómeno en la teoría de los nuevos movimientos sociales (NMS) expuesta, entre otros, por Alain Touraine, Manuel Castells, Jürgen Habermas y la academia crítica posmoderna. En muchas de sus formulaciones teóricas, los NMS son presentados como respuestas específicas a la cultura hegemónica y totalizadora definida por los mercados capitalistas y prestan especial atención al papel del consumidor (Kozinets y Handelman, 2004). 


\section{EL CONSUMO POLÍTICO EN ARGENTINA: ESTRATEGIAS DESARROLLADAS POR ORGANIZACIONES SIN FINES DE LUCRO}

El uso del mercado como un lugar para la participación política no es un fenómeno nuevo. Los bienes de consumo se utilizaron como elemento movilizador contra la esclavitud (finales del s. XVIII), en la lucha por la independencia de la India (s. XIX-XX) o en las protestas internacionales contra la guerra de Vietnam y el apartheid surafricano (estos tres últimos en la segunda mitad del s. XX), entre otros (Micheletti, 2002).

No obstante, el consumo político no ha despertado particular interés entre académicos, líderes empresariales y políticos hasta principios de los 90 (Low y Davenport, 2007). Actualmente es un movimiento en continuo crecimiento en las democracias industriales avanzadas, especialmente visible en los países escandinavos (Stolle y Micheletti, 2005).

Según la Encuesta Social Europea (ESE) de 2002 , el 16,5\% de la población había boicoteado mediante sus compras durante el último año. En Suiza y Suecia el porcentaje superó el 32\%, y Finlandia y Reino Unido se situaron por encima del 26\%. En la ESE de 2002 también se consultó a los ciudadanos si habían comprado algún producto por razones políticas, éticas o medioambientales ${ }^{2}$. El $26,3 \%$ respondió afirmativamente, destacando los casos de Suecia, Suiza, Dinamarca y Finlandia, por encima del $41 \%$. En la última ESE realizada en 20143 , el $23 \%$ de la población había boicoteado durante el último año, un 6,5\% más que en 2002. Destacan los casos de Suecia, Alemania y Finlandia (más del $36 \%$ ), en contraste con Polonia, Eslovenia y Estonia (por debajo del 8\%) (ESE, 2015).

Según los estudios contemporáneos, las mujeres desempeñan un rol destacado en la práctica del movimiento, especialmente en Europa Occidental, y los valores postmaterialistas aparecen como el factor más explicativo del comportamiento del consumidor político (Micheletti, 2002; Stolle y Micheletti, 2005). El perfil lo completan características entre las que sobresalen: juventud, nivel educativo y nivel de ingresos alto, cosmopolitismo, desconfianza hacia las instituciones, interés por la política y pertenencia a grupos o asociaciones, entre otras. Si bien en casi todos los estudios se confirman las mismas relaciones entre variables, conviene señalar que difieren en función del país analizado (Novo Vázquez, 2014).

Por otro lado, el consumo político guarda una estrecha relación con la Responsabilidad Social Corporativa (RSC), que en el ámbito del desarrollo es una herramienta de sostenibilidad y legitimidad social de la empresa, así como de mejora de leyes y condiciones institucionales de un país. El comportamiento de los consumidores puede empujar a las empresas a la gestión responsable de su cadena de valor. En gran medida, las empresas producen lo que los consumidores demandan, por tanto éstos pueden enviar señales al mercado que fomenten la adopción de prácticas y modelos de gestión más responsables.

1.- Realizada a 22 países europeos, entre los cuales se incluyó a Israel.

2.- En las siguientes encuestas, la última de ellas realizada en 2014, no se repitió esta pregunta.

3.- La consulta incluyó a 15 países europeos. 


\subsection{Teoría institucional y estrategias de cambio basadas en el consumo político que pueden realizar las organizaciones}

La creciente influencia de las OSFL ha sido un hecho destacado a nivel internacional desde los 80. Su capacidad para obtener legitimidad depende en parte de las estructuras institucionales y el legado político de la región donde actúan (Doh y Guay, 2006).

Campbell (2007) expone claramente las condiciones que influyen a las empresas para ser socialmente responsables. Por un lado, condiciones económicas, que comprenden la situación financiera relativa de la empresa y de la economía y el nivel de competencia. Por otro lado, condiciones institucionales que afectan a las anteriores, tales como la regulación estatal, la auto regulación colectiva, el compromiso con la RSC desde el entorno institucional normativo y por parte de las asociaciones de empresarios, la participación en un diálogo institucionalizado con stakeholders y, la más importante para nuestro caso, la existencia de organizaciones privadas e independientes, como OSFL, inversores institucionales o medios de comunicación que vigilen su comportamiento y se movilicen para cambiarlo cuando sea necesario.

Las OSFL pueden jugar un papel muy importante a la hora de enfrentar los fallos de mercado apoyándose en la RSC (Pinzón Camargo, 2005). En el caso del consumo político, el funcionamiento imperfecto del mercado exige grandes esfuerzos personales de información y decisión para hacer frente a dos importantes fallos (Valor, 2011):

- Asimetría informativa: la empresa conoce su desempeño social y ambiental pero el consumidor, en la mayoría de los casos, no.

- Asimetría de poder: las acciones del consumidor tienen un impacto limitado en la empresa, y ésta tiene más capacidad para dictar los criterios de elección.

Ambas asimetrías sitúan al consumidor político como un sujeto a contracorriente y en un papel un tanto heroico, especialmente si se halla desprovisto de plataformas e incentivos para la movilización. Ahí radica la importancia de las OSFL por su capacidad de movilización social, esto es, por constituir redes de reciprocidad, cooperación voluntaria y compromiso, permitiendo la interrelación entre individuos en estructuras capaces de articular la acción colectiva. En nuestro caso, como intermediarias y aglutinadoras de esfuerzos conjuntos para empoderar a los consumidores frente al mercado (Vargas, 2003; Novo Vázquez, 2014).

Sería posible resumir la labor de las OSFL en su conjunto en cinco verbos: apoyar, vigilar, defender, promover e incomodar. Cabe distinguir a las OSFL como una de las fuerzas de ampliación del espacio público y de fortalecimiento de las sociedades civiles (Grzybowski, 2001).

En este contexto, siguiendo a Bendell (2007), la sociedad civil -conformada por grupos civiles, que son organizaciones y redes formales o informales, sin ánimo de lucro y no gubernamentales-, dispone 


\section{EL CONSUMO POLÍTICO EN ARGENTINA: ESTRATEGIAS DESARROLLADAS POR ORGANIZACIONES SIN FINES DE LUCRO}

de una herramienta para impulsar la democratización de la actividad económica: la regulación civil. Esta herramienta reconoce a la sociedad civil como agente para el debate sobre las normas de comportamiento de las corporaciones. Así, los grupos civiles resuelven acerca de los estándares que deberían ser mantenidos por éstas como sujetos de la regulación. Sus resoluciones son diversas, toman la forma de objetivos de campañas, códigos de conducta o sistemas de certificación. El marco regulatorio es completado por los mecanismos de evaluación del cumplimiento.

Bendell (2007) propone una tipología de regulación civil basada en dos variables (ver tabla 1): lugar. dentro o fuera del mercado, dependiendo de si se buscan beneficios económicos o no; y estilo: cooperación o conflicto, dependiendo de si la relación establecida es de colaboración o de confrontación:

\section{Tabla 1. Tipos de estrategias dirigidas a influenciar sobre las corporaciones}

\begin{tabular}{|l|c|c|}
\hline & Cooperación & Conflicto \\
\hline Fuera del mercado & Promoviendo el cambio & Forzando el cambio \\
\hline Dentro del mercado & Facilitando el cambio & Produciendo el cambio \\
\hline
\end{tabular}

FUENTE: Elaborado a partir de Bendell, 2007.

- Promoviendo el cambio: los grupos civiles pueden donar su tiempo, sus recursos y su dinero para acciones tales como el diálogo entre empresas, consultores y grupos civiles, apoyar las mejores prácticas y promocionarlas, negociar acuerdos, dirigir y publicar investigaciones útiles o desarrollar conjuntamente nuevos productos, técnicas o procedimientos.

- Facilitando el cambio: los grupos civiles pueden vender servicios como la consultoría en materia de estrategia, política y cambio organizacionales o la gestión de redes o instituciones que realicen promoción, certificación o acreditación de estándares de sostenibilidad, mejorando de este modo la reputación de su cliente.

- Forzando el cambio: a nivel activista, como forma de presión, los grupos civiles pueden organizar boicots, exponer cuestiones incómodas a través de los medios de comunicación, manifestarse en tiendas, oficinas o juntas de accionistas y movilizar a la opinión pública en contra de una empresa determinada.

- Produciendo el cambio: los grupos civiles pueden proveer medios de producción alternativos y sistemas de comercialización basados en sistemas de valores diferentes de los del mercado mainstream, como el comercio justo o la agricultura orgánica.

Desde el punto de vista de las OSFL, la colaboración con las empresas puede resultar necesaria para alcanzar sus objetivos pero también paradójica, ya que a su vez crea tensiones que pueden socavar los objetivos del movimiento (Bendell, 2004). 
Los gobiernos también pueden asumir un rol activo para lograr cambios en el mercado (Micheletti y Follesdal, 2007). Es el caso de los "espacios éticos", que pueden ser legislados, donde se induce a la toma de decisiones responsables, por ejemplo, gravando con un impuesto las bolsas de plástico no biodegradables; o voluntarios, mediante la adopción de compromisos que sirven de empuje, como las ciudades por el comercio justo (Low y Davenport, 2007).

La tipología presentada arriba será retomada en la sección de resultados para analizar las estrategias sobre consumo político llevadas a cabo por las OSFL argentinas, respondiendo así a la pregunta de investigación propuesta.

Después de encuadrar el fenómeno desde el punto de vista de las organizaciones en su rol de movilizadoras del consumo político, a continuación pondremos el foco en el consumidor individual, en su rol de activista para la regulación civil mediante el consumo político.

\subsection{Acciones que puede realizar el consumidor}

Para entender las actos de consumo o no consumo antes mencionados, siguiendo a Papaoikonomou (2012) y Valor (2011), podemos distinguir cuatro grandes categorías de acciones de consumo político desde el punto de vista del consumidor (ver tabla 2):

Tabla 2. Tipos de acciones de los consumidores políticos

\begin{tabular}{|l|c|c|}
\hline & Convencional (mainstream) & Alternativo \\
\hline Comprando & Buycotting & Smart shopping \\
\hline No comprando & Boycotting & Simplifiying \\
\hline
\end{tabular}

FUENTE: Elaborado a partir de Valor, 2011: 12.

a) Boycotting: categoría con mayor recorrido histórico dentro del movimiento (Low y Davenport, 2007). Son actos de consumo negativo a través de los cuales los consumidores manifiestan sus preocupaciones sociales evitando ciertas marcas, no comprando determinados productos, presionando a empresas o tratando de movilizar al resto de consumidores en la misma dirección. Existen distintos tipos de boicots que pueden agruparse según su objeto, función 0 acción solicitada.

b) Buycotting: acciones consistentes en elegir y comprar mediante canales convencionales ciertos productos, servicios y marcas en lugar de otros debido a consideraciones éticas, sociales y ambientales. Por lo tanto, en contraste con el boicot, se trata de un consumo positivo. 


\section{EL CONSUMO POLÍTICO EN ARGENTINA: ESTRATEGIAS DESARROLLADAS POR ORGANIZACIONES SIN FINES DE LUCRO}

c) Smart shopping: los actos de consumo inteligente son aquellos realizados a través de canales alternativos que tienen como objetivo transformar las estructuras de mercado, como el comercio justo o la banca ética.

d) Simplifying: los simplificadores voluntarios o éticos eligen reducir su nivel de consumo para adoptar un estilo de vida más simple porque consideran que el modelo social que promueve el materialismo y el consumismo es ambiental y socialmente insostenible. Su repertorio de prácticas se enmarca en el modelo 3R (triple "r"): reciclar, reducir y reutilizar.

Estas categorías servirán para analizar, en la sección de resultados, las alternativas para el consumidor político sobre las que inciden las OSFL de Argentina.

La amplia variedad de acciones expuesta refleja la diversidad y el pluralismo del movimiento (Klintman, 2006). Los consumidores políticos tienen diferentes concepciones de lo que es ético, así como diferentes posibilidades de actuación, y esto se ve reflejado en su posicionamiento ante el mercado y en sus pautas de compra. De acuerdo con el trabajo de Papaoikonomou (2012) distinguimos dos perspectivas en conflicto. La primera concibe el consumo como una vía de participación política, como una forma de votar en el mercado mediante sus actos de compra o no compra, premiando y castigando a las empresas en función de su responsabilidad social. De este modo, el poder de compra se emplea como una nueva herramienta democrática y como palanca de cambio.

La segunda perspectiva sitúa al consumo, incluso al ético, como parte del problema debido a su constante crecimiento. Gran parte de las acciones de consumo responsable no apuntan a resolver el origen de los problemas reales: capitalismo, materialismo, crisis medio ambiental y pobreza. Lo ético no es mantener o aumentar el nivel de consumo sino reducirlo en sentido amplio.

Otra cuestión a tener en cuenta respecto del comportamiento del consumidor es la llamada brecha ética, definida como la diferencia entre el porcentaje de consumidores que dicen que comprarían productos éticos y aquellos que realmente los compran. Las razones que pueden explicar esta brecha son: falta o exceso de información, gama de productos y disponibilidad limitadas, precios más elevados, mayor tiempo de búsqueda, falta de compromiso y sentimiento de que la acción propia no marca la diferencia (Low y Davenport, 2007). 


\section{3.- Contexto del consumo político en Argentina}

El contexto argentino del consumo político está formado por diversos factores que serán abordados en esta sección: la historia del sector sin fines de lucro; factores de mercado y políticos; y las características actuales del sector sin fines de lucro.

El 29\% de los argentinos considera que la manera más efectiva de conseguir cambios en su país es presionando como consumidor para que las empresas sean más responsables social y ambientalmente, en contraste con solo el $15 \%$ en México y el $19 \%$ en Brasil ${ }^{4}$. Que casi un tercio de los argentinos confíe en el poder de los consumidores puede resultar menos sorprendente si tenemos en cuenta la línea temporal de la figura 1, que muestra la evolución del sector sin fines de lucro en el país desde la época colonial hasta la actualidad. A mediados de siglo XX, la creación de la Fundación Eva Perón ya introdujo la noción de justicia social. El retorno a la democracia (1983) dio inicio a un importante desarrollo del sector sin fines de lucro que, unido a la penetración de las empresas transnacionales en los 90, propició que se comenzara a hablar de RSC (Paladino y Mohan, 2002). En la década del 2000, especialmente como respuesta reactiva a la situación de crisis, emergieron emprendimientos de economía social y movimientos de economía solidaria y consumo político (Mateos y Ghezán, 2010; Saguier, 2005).

\section{Figura 1. Evolución temporal del sector sin fines de lucro argentino}

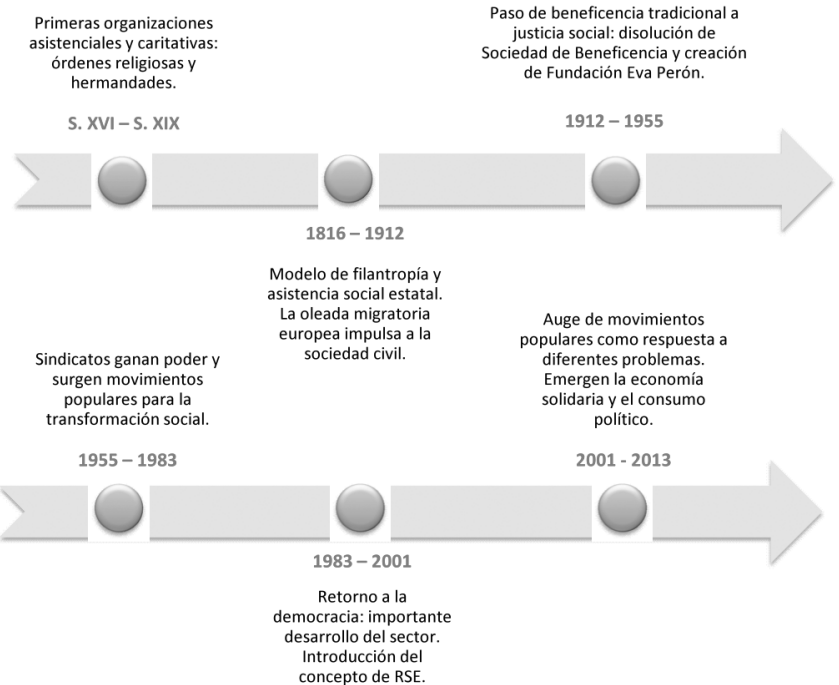

FUENTE: Elaboración propia. 


\section{EL CONSUMO POLÍTICO EN ARGENTINA: ESTRATEGIAS DESARROLLADAS POR ORGANIZACIONES SIN FINES DE LUCRO}

Otros factores que condicionan el contexto del consumo político en Argentina pueden ser observados en la figura 2, que es una adaptación ${ }^{5}$ del modelo de Thogersen (2010). Este autor afirma que los factores macro y estructurales condicionan el nivel de sostenibilidad del consumo, ya que impactan fuertemente en las decisiones individuales de los consumidores finales.

\section{Figura 2. Determinantes macro del consumo político}

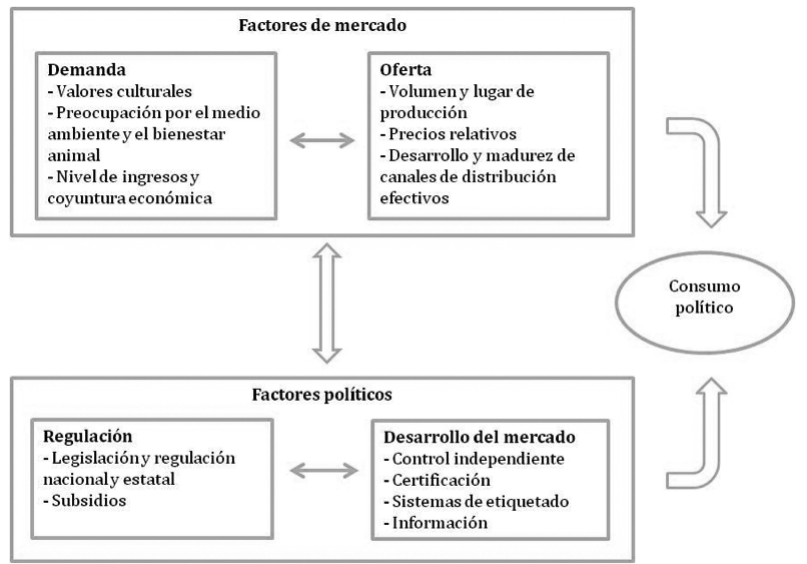

FUENTE: Elaborado a partir de Thogersen, 2010: 176.

En relación con los factores de mercado podemos destacar varias características:

Según la teoría sobre el cambio de valores en los países occidentales formulada por Inglehart (1977 citado en Corbetta, 2007: 73-74), el paso de la orientación materialista a la postmaterialista depende de las condiciones de vida durante los años de formación de los ciudadanos, y el número de personas que abrazan los valores postmaterialistas debería ser mayor cuanto más rico sea el país.

Argentina entró recientemente al grupo de países de ingreso alto (Banco Mundial, 2015) y se sitúa en el puesto 49 de 187 en el índice de desarrollo humano (IDH). Si bien se encuentra en el grupo de IDH muy alto, con un valor de 0,808, se mantiene a una distancia considerable del promedio del grupo $(0,890)$ y cae a 0,680 si se ajusta teniendo en cuenta la desigualdad (PNUD, 2014: 194-198). De

4.- Encuesta realizada por Market Analysis y GlobeScan, Monitor de Responsabilidad Social Corporativa. Los datos provienen de muestras urbanas realizadas en Brasil (noviembre 2008), Argentina (enero 2009) y México (julio 2009). En Argentina fueron entrevistados 410 adultos de forma telefónica, en el área metropolitana de Buenos Aires, Rosario ciudad y Córdoba ciudad, a partir de un sorteo aleatorio de números, ajustando la muestra final según cuotas de sexo, edad y clase, con casos por ciudad distribuidos proporcional al tamaño de la población (Echegaray y Sarsfield, 2010).

5.- El modelo de análisis de los condicionantes estructurales del consumo de alimentos orgánicos en Europa ha sido extrapolado a nuestro caso de estudio. Se mantuvieron las categorías factores de mercado y factores políticos pero se modificaron algunos de los factores que allí se incluyen, de forma que el modelo abarcara al consumo político en su conjunto y evitara las particularidades europeas. 
acuerdo con estos indicadores, podemos decir que Argentina no se encuentra entre los países donde se manifestarían en mayor medida los valores postmaterialistas.

Por otro lado, un punto a tener en cuenta sobre la oferta para consumidores políticos es el elevado volumen de producción orgánica (agrícola y ganadera) en suelo argentino, entre los más altos del mundo según el Movimiento Argentino para la Producción Orgánica (2013). Sin embargo, dicha producción está fuertemente orientada a la exportación y el mercado interno tiene poca relevancia. Más del 95\% de los alimentos orgánicos producidos se dirige al mercado externo y, en 2013, la participación de productos certificados en el mercado doméstico fue del 1\% (Mateos y Ghezán, 2010; Senasa, 2014).

También cabe destacar que, en el caso de la producción bajo criterios de comercio justo, el continente latinoamericano es altamente exportador (Coscione, 2008). Argentina sigue esta dinámica con un mercado interno muy poco desarrollado, aunque no existen datos acerca de su evolución (Fundación Fortalecer, 2012; Mateos y Ghezán, 2010).

En relación con el ámbito político, los factores más relevantes detectados para favorecer el consumo político son:

- En el aspecto legislativo:

a) La existencia de la Dirección de Producción Limpia y Consumo Sustentable, dependiente de la Secretaría de Ambiente y Desarrollo Sustentable de la Nación.

b) La Ley 25.127 - Producción ecológica, biológica u orgánica de 1999, de ámbito estatal.

c) Promoción de compras públicas sostenibles por parte del Gobierno nacional, a través de formación y asistencia técnica.

d) Política de promoción del consumo responsable del Gobierno de la ciudad de Buenos Aires.

- En lo referido a certificación y sistemas de etiquetado, además de la presencia en el país de sellos internacionales reconocidos como los de Forest Stewardship Council (FSC) o Fairtrade, encontramos dos iniciativas estatales, la etiqueta de eficiencia energética y el reciente sello "Orgánico Argentino", además de varias iniciativas nacionales privadas. No hay disponibles cifras de ventas totales o de cuota de mercado de productos y establecimientos certificados.

Tras este análisis, a partir de la escasa información disponible podría deducirse un débil desarrollo de oferta y demanda; mientras que entre los factores políticos encontramos iniciativas positivas de las que desconocemos su impacto pero, en apariencia, carecen de recorrido y solidez, a excepción de la ley de producción ecológica.

Por último, el contexto argentino del consumo político estaría influido por las características del sector sin fines de lucro, que veremos a continuación.

En 2004 existían en Argentina 104.642 organizaciones pertenecientes al sector sin fines de lucro (Luna y Cecconi, 2004), el cual tiene una importante participación tanto en la población activa como 


\section{EL CONSUMO POLÍTICO EN ARGENTINA: ESTRATEGIAS DESARROLLADAS POR ORGANIZACIONES SIN FINES DE LUCRO}

en el PIB (Center for Civil Society Studies, 2004). Las OSFL "tienen una significación de 2,9 organizaciones cada mil habitantes, colocándose Argentina por encima de varios países de América Latina (Chile 2,8 - Brasil 0,7) y por debajo de Estados Unidos (4,30), y de muchos países de Europa Occidental (España 3,8 - Gran Bretaña 5,1 - Francia 12,1)" (Luna y Cecconi, 2004: 20).

En la figura 3 puede observarse que las áreas temáticas de mayor relevancia son las de un perfil solidario más tradicional, mientras que las cuestiones emergentes, propias de las organizaciones de advocacy, tienen una presencia mucho menor (Luna y Cecconi, 2004). De hecho, los subsectores que mayoritariamente están conformados por entidades que producen servicios intangibles, tales como la promoción de derechos humanos y la defensa del medio ambiente, tienen un menor peso en el empleo y en la generación del PIB (Roitter y González Bombal, 2000).

\section{Figura 3. Áreas temáticas atendidas por las OSFL en Argentina}

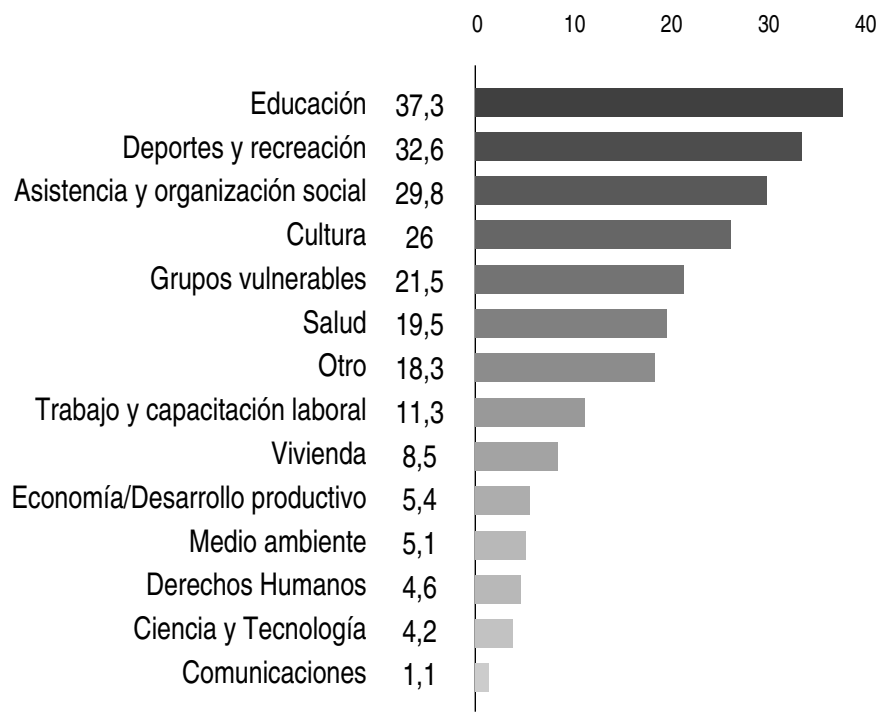

FUENTE: Luna y Cecconi, 2004: 28.

Hecho este análisis, puede inferirse que a pesar del notable desarrollo del Tercer Sector argentino, no existiría una fuerte presencia de OSFL que tengan entre sus objetivos el fomento del consumo político.

Por tanto, podemos concluir que, si bien el contexto argentino no es adverso, dista de presentar las condiciones de aquellos países donde el movimiento está más desarrollado. 


\section{4.- Metodología}

La metodología elegida para esta investigación es el estudio de casos, particularmente apropiada para los fenómenos nuevos o de los que se sabe poco, en donde la teoría se encuentra en sus primeras fases de desarrollo, así como para analizar las experiencias que se dan en las organizaciones (Castro Monge, 2010; Eisenhardt, 1989).

En este sentido, podemos considerar adecuada la elección del estudio de casos para la presente investigación ya que, si bien el fenómeno del consumo político no es de nuevo origen, su difusión práctica e interés académico sí son recientes. Asimismo, se estudia su desarrollo actual en Argentina, un escenario apenas analizado.

La población objeto de estudio es el conjunto de OSFL presentes físicamente en Argentina, ya sean nacionales 0 internacionales, siempre que tengan al menos una sede y/o un equipo de trabajo en el país. Siguiendo la definición de Salamon y Anheier (1992 en Roitter y González Bombal, 2000: 16), establecemos cinco criterios que deben cumplir las OSFL para ser consideradas como tales en este trabajo: estructuradas, sin fines de lucro, privadas, autogobernadas y de libre afiliación.

Además, para formar parte de la población, las OSFL deben cumplir otros dos requisitos: defensa de un modelo de desarrollo sostenible como eje central de su actividad; y presencia del consumo político 0 uno de sus tipos de acciones como línea de trabajo u objetivo.

La elección de los casos no se ha realizado según criterios muestrales estadísticos sino por razones teóricas. Hemos pretendido obtener un conjunto diverso y conceptualmente representativo, buscando deliberadamente diferencias entre casos para alcanzar la saturación teórica (Eisenhardt, 1989), con base en cinco criterios: tipo de OSFL según su ámbito de actividad, tamaño, ámbito territorial y los dos criterios excluyentes expuestos en el párrafo de arriba. El muestreo teórico resultante debe consultarse en la tabla 3 . El conjunto de las seis OSFL, su suma total, constituye nuestro estudio de caso dentro del campo de las OSFL en este país, ya que con ellos se alcanza el punto de saturación teórica. 


\section{Tabla 3. Organizaciones objeto de estudio y criterios empleados}

\begin{tabular}{|c|c|c|c|c|c|c|}
\hline Nombre completo & $\begin{array}{c}\text { INSTITUTO } \\
\text { ARGENTINO } \\
\text { PARA EL } \\
\text { DESARROLLO } \\
\text { SUSTENTABLE } \\
\text { (IADS) }\end{array}$ & $\begin{array}{c}\text { ASOCIACIÓN } \\
\text { CIVIL } \\
\text { PORLA } \\
\text { RESPONSAB. } \\
\text { SOCIAL } \\
\text { AMARTYA }\end{array}$ & $\begin{array}{l}\text { CONSUMIDORES } \\
\text { ARGENTINOS }\end{array}$ & $\begin{array}{c}\text { FUNDACIÓN } \\
\text { VIDA } \\
\text { SILVESTRE } \\
\text { ARGENTINA }\end{array}$ & $\begin{array}{c}\text { FUNDACIÓN } \\
\text { FORTALECER }\end{array}$ & $\begin{array}{c}\text { INSTITUTO } \\
\text { PARA EL } \\
\text { COMERCIO } \\
\text { EQUITATIVO } \\
\text { YEL CONSUMO } \\
\text { RESPONSABLE } \\
\text { (Icecor) } \\
\end{array}$ \\
\hline Forma jurídica & Asociación civil & Asociación civil & Asociación civil & Fundación & Fundación & Asociación civil \\
\hline $\begin{array}{l}\text { Tipo de OSFL } \\
\text { (ámbito actividad) }\end{array}$ & Think tank & $\begin{array}{c}\text { Educación para } \\
\text { el desarrollo }\end{array}$ & $\begin{array}{l}\text { Defensa del } \\
\text { consumidor }\end{array}$ & Medioambiental & Desarrollo rural & Think tank \\
\hline Tamaño & Pequeño & Pequeño & Pequeño & Mediano & Pequeño & Pequeño \\
\hline Ámbito territorial & Nacional & Nacional & $\begin{array}{c}\text { Nacional } \\
\text { (miembro Consumers } \\
\text { International) }\end{array}$ & $\begin{array}{c}\text { Nacional } \\
\text { (miembro WWF } \\
\text {-World Wildlife Fund-) }\end{array}$ & Nacional & Nacional \\
\hline $\begin{array}{l}\text { Desarrollo sostenible } \\
\text { (como eje central) }\end{array}$ & Qué hacemos & Misión y visión & Objetivos & Misión y visión & Misión & Quiénes somos \\
\hline $\begin{array}{l}\text { Consumo político } \\
\text { (como línea de trabajo } \\
\text { u objetivo) }\end{array}$ & Central & Central & Central & Periférico & Central & Central \\
\hline
\end{tabular}

FUENTE: Elaboración propia.

Para dotar de validez a la investigación, se obtuvieron datos de diferentes fuentes que convergieran en un estilo de triangulación (Eisenhardt, 1989). Para garantizar la fiabilidad (Castro Monge, 2010), presentamos el protocolo seguido para los seis casos. En primer lugar, para la localización de las OSFL radicadas en Argentina se emplearon las siguientes fuentes y recursos: web de OSFL y de redes organizativas, memorias anuales, estatutos e informes contables, perfil en redes sociales y método de muestreo snowball / referral sampling.

En segundo lugar, una vez localizadas las OSFL, las fuentes previstas para detectar, describir y encuadrar las estrategias sobre consumo político fueron: indagación y análisis minucioso de webs, memorias anuales y perfiles en redes sociales; entrevista telefónica ${ }^{6}$ a los referentes de las OSFL (semiestructurada en base a un guión ligado a las variables de estudio y a la revisión de la literatura, ver tabla 4) y referencias en medios de comunicación. En la tabla 5 puede observarse la lista de personas entrevistadas y, en la tabla 6 , las iniciativas desarrolladas por cada OSFL.

\section{6.- Excepto a Vida Silvestre, que no accedió a conceder la entrevista telefónica, sino mediante correo electrónico.}




\section{Tabla 4. Variables y preguntas de investigación}

\begin{tabular}{|c|c|c|}
\hline Variables & Preguntas del guión & Fuentes \\
\hline Contexto & $\begin{array}{l}\text { 1.1. ¿Cómo ve la situación del consumo político en Argentina? } \\
\text { 1.2. ¿Cuáles cree que son las principales oportunidades y limitaciones del } \\
\text { contexto argentino? }\end{array}$ & $\begin{array}{c}\text { Echegaray y Sarsfield, 2010; } \\
\text { referencias del punto } 3\end{array}$ \\
\hline Estrategia & $\begin{array}{l}\text { 2.1. ¿Por qué está el consumo político en sus objetivos y desde cuándo? } \\
\text { 2.2. ¿Cuántas campañas o proyectos de fomento del consumo político han } \\
\text { realizado en los últimos tres años? } \\
\text { 2.3. Describa las tres principales: } \\
\text { 2.3.1. ¿En qué consistieron? } \\
\text { 2.3.2. ¿Cuáles fueron las actividades realizadas? } \\
\text { 2.3.3. Duración y localización geográfica. } \\
\text { 2.3.4. Presupuesto y personal atribuidos. } \\
\text { 2.3.5. ¿Por qué las realizaron? Justificación estratégica. }\end{array}$ & $\begin{array}{l}\text { Bendell, 2007; } \\
\text { Papaoikonomou, 2012; } \\
\quad \text { Valor, 2011; } \\
\text { Valor y Merino, } 2009\end{array}$ \\
\hline Resultados & $\begin{array}{l}\text { 3.1. Evaluación y resultados obtenidos. } \\
\text { 3.2. ¿Cuál fue la respuesta de las personas y/o las organizaciones a las que } \\
\text { dirigieron las campañas? } \\
\text { 3.3. ¿Qué aprendió su organización tras/durante la realización de estas campañas? }\end{array}$ & $\begin{array}{c}\text { Bendell, 2004; } \\
\text { Klintman, 2006; } \\
\text { Low y Davenport, } 2007\end{array}$ \\
\hline
\end{tabular}

FUENTE: Elaboración propia.

\section{Tabla 5. Referentes entrevistados}

\begin{tabular}{|lcc|}
\hline IADS & Tesorero y socio fundador & A \\
Amartya & Director Ejecutivo & B \\
Consumidores argentinos & Presidenta & C \\
Vida Silvestre & Coordinador de Comunicación & D \\
Fortalecer & Coordinadora técnica Programa Comercio Justo & E \\
Icecor & Presidente & $\mathrm{F}$ \\
\hline
\end{tabular}

FUENTE: Elaboración propia. 


\section{Tabla 6. Iniciativas desarrolladas por cada OSFL}

\begin{tabular}{|l|l|}
\hline OSFL & Iniciativa \\
\hline IADS & $\begin{array}{l}\text { a. Construyendo comunidades más sustentables } \\
\text { b. Compras públicas sustentables } \\
\text { c. Producción sustentable }\end{array}$ \\
\hline Amartya & $\begin{array}{l}\text { a. Consuma dignidad } \\
\text { b. Semillas de sustentabilidad } \\
\text { c. Vídeos de concienciación }\end{array}$ \\
\hline Consumidores Argentinos & $\begin{array}{l}\text { a. Alimentos orgánicos, más salud para todos } \\
\text { b. Yo traigo mi bolsa } \\
\text { c. Membresía en PERL (Partnership for education and research about responsible living) }\end{array}$ \\
\hline Fortalecer & $\begin{array}{l}\text { a. La Hora del Planeta } \\
\text { b. Membresía en FSC } \\
\text { c. Campaña Merluza }\end{array}$ \\
\hline Icecor & $\begin{array}{l}\text { a. Promoción de Sistemas Productivos Sustentables a través de Mercados de Comercio Justo } \\
\text { b. Diseño, gestión y evaluación de proyectos }\end{array}$ \\
\hline
\end{tabular}

FUENTE: Elaboración propia.

\section{5.- Resultados}

\subsection{Valoración de las OSFL del contexto argentino para el consumo político}

Todos los entrevistados coinciden en que es un movimiento muy poco desarrollado en el país. Mayormente, sitúan las causas en los factores de mercado, apuntando especialmente a la escasez de demanda.

"Existe una brecha entre la buena intención y la compra efectiva. Además, no hay educación en consumo político ni en derechos del consumidor". (C) 
"Hay una gran impronta de las soluciones individuales a los problemas, que proviene de una formación política liberal, individualista. No se consigue un consumidor activista que resigne gustos 0 comodidades". (F)

Aunque en menor medida, también se señalan problemas de oferta.

"Las iniciativas son de poca escala, orientadas a un perfil particular y no al consumidor masivo". (A) "Los productos responsables tienen altos precios". (E)

Los factores políticos, centrados en la escasa regulación estatal, fueron menos ponderados como causa de este débil desarrollo.

Por otra parte, varios entrevistados destacaron la ausencia de estadísticas, necesarias para evaluar acertadamente el movimiento.

En cuanto a las oportunidades para la expansión del consumo político, la mayoría de entrevistados apunta que el interés y la conciencia de los consumidores va en aumento, por tanto existe un potencial de crecimiento que ya se está materializando en ferias de productores, nuevos emprendimientos y presencia en los medios de comunicación, entre otros.

(El tema) "Está en la agenda pública". (C)

5.2. Análisis global de las principales estrategias desarrolladas por las OSFL

- ¿Cuáles son las estrategias de cambio utilizadas para fomentar el consumo político?

En este punto encontramos dos hechos sobresalientes. El primero, las estrategias basadas en la cooperación son abrumadoramente mayoritarias. El segundo, el lugar de actuación predominante es fuera del mercado (ver tabla 7). 


\section{Tabla 7. Tipos de estrategias de cambio desarrolladas por el conjunto de OSFL analizadas}

\begin{tabular}{|l|l|l|}
\hline & Cooperación & Conflicto \\
\hline \multirow{4}{*}{ Fuera del mercado } & Promoviendo el cambio & Forzando el cambio \\
& - IADS a., b. y c. & - Vida Silvestre c. \\
& - Amartya a., b. y c. & \\
& - Consumidores Argentinos a., b. y c. & \\
& - Vida Silvestre a. y b. & \\
& - Fortalecer a. & \\
& - Icecor a. & Produciendo el cambio \\
\hline \multirow{3}{*}{ Dentro del mercado } & Facilitando el cambio & - Fortalecer a. y b. \\
& - IADS b. y c. & - Icecor b. y c. \\
\hline
\end{tabular}

FUENTE: Elaborado a partir de Bendell, 2007.

De esta manera, cabe afirmar que las OSFL prefieren el estilo colaborativo en su relación con empresas y administraciones públicas. Todas ellas pusieron en práctica alguna estrategia de cooperación. Es más, solo tres organizaciones basaron en el conflicto alguno de sus programas o campañas, o parte de ellos; el resto, ninguno. Decimos parte porque un mismo programa puede contener varias estrategias diferentes.

Del mismo modo, podemos decir que la mayoría no pretende la recaudación de fondos con sus estrategias. Todas las OSFL pusieron en práctica alguna iniciativa (o al menos parte de ella, en el caso de Fortalecer) fuera del mercado.

Como consecuencia de los dos hechos anteriores, encontramos un peso muy significativo de las estrategias que promueven el cambio, es decir, que están basadas en la cooperación y ubicadas fuera del mercado, por no pretender la obtención de beneficios económicos para la propia OSFL. Entre la variedad de acciones promotoras del cambio planteadas por Bendell (2007) (ver en 2.2.), las más recurrentes son el asesoramiento y la difusión de buenas prácticas.

Siguiendo con las relaciones basadas en la cooperación, ahora dentro del mercado, observamos que el cuadrante conformado por las iniciativas facilitadoras del cambio tiene un peso mucho menor que el de las promotoras del cambio. Aquí encontramos diversos servicios ofrecidos por IADS en dos de sus programas, que ofrecen asistencia técnica, formación y consultoría. 
En cuanto a las relaciones de conflicto, esto es, aquellas que confrontan con las corporaciones mainstream, observamos menor saturación de estrategias. Fuera del mercado, tan sólo una de Vida Silvestre, cuyo fin es forzar el cambio exponiendo una cuestión incómoda a través de los medios de comunicación y movilizando a la opinión pública en contra de un producto determinado, la merluza de pequeño tamaño. El resto, dentro del mercado, buscan producir el cambio proveyendo una herramienta de producción (arado ecológico de Icecor) y sistemas de comercialización (para comercio justo desde Fortalecer; para la agricultura ecológica y la economía social desde Icecor) alternativos a los del mercado convencional.

En cuanto a los motivos de la implementación de estas últimas estrategias, no encontramos un patrón común para las tres OSFL, sin embargo podemos aproximarnos a ellos de forma individual:

- Vida Silvestre es una organización de advocacy-defensora del medio ambiente-, cuenta con la mayor base social entre las analizadas y probablemente es la más popular y mediática. Así pues, reúne las características oportunas para ejercer presión interpelando a la opinión pública.

- Icecor se distingue por ser la OSFL más crítica con las fuerzas del mercado en relación con la inequidad que generan y por ello promueve un esquema de relaciones comerciales diferente y superador. De ahí que resulten coherentes sus actividades de fomento de la agricultura ecológica, el comercio justo y la economía social.

- En el caso de Fortalecer no observamos una crítica abierta al sistema económico dominante, si bien el comercio justo encaja perfectamente en su misión de promover el desarrollo rural sostenible y equitativo, especialmente cuando trabaja con pequeños productores.

Es importante aclarar que, a pesar de que en diferentes casos las OSFL anteriores no establecen una relación de confrontación directa con la otra parte (por ejemplo, productores agropecuarios), sus acciones sí entran en conflicto con el mercado y las corporaciones convencionales, y por ende, con el sistema neoliberal, de ahí que se ubiquen en este cuadrante.

Por otro lado, podemos destacar varios aspectos de las tres OSFL que basan todas sus estrategias en la cooperación. IADS y Amartya incluyen en su misión el trabajo junto con la sociedad civil y los sectores público y privado, lo que podría inclinarlos a ejercer un rol de mediación o articulación y no tanto de confrontación. Consumidores Argentinos y Amartya presentan un importante componente pedagógico en su identidad, también manifestado explícitamente en su misión. La primera es un claro caso de OSFL de advocacy, sin embargo su pequeña envergadura podría motivar que no se decante por emprender acciones "cuerpo a cuerpo" contra el poder corporativo sino por la conformación de una masa social crítica a través de la difusión de buenas prácticas, en línea con su misión educativa. 
- ¿Cuáles son las alternativas sobre las que inciden las OSFL en relación con las acciones que puede realizar el consumidor?

A diferencia de las estrategias de cambio, observamos una mayor saturación de los cuadrantes y una presencia más abarcadora de las organizaciones (ver tabla 8). Salvo en el caso de Icecor, que se concentra en smart shopping, el resto actúa al menos en dos cuadrantes. Destacan Consumidores Argentinos y Vida Silvestre, que inciden en los cuatro grupos de acciones.

\section{Tabla 8. Tipos de acciones sobre las que inciden las OSFL analizadas}

\begin{tabular}{|l|l|l|}
\hline & Cooperación & Conflicto \\
\hline & Buycotting & Smart shopping \\
& - IADS a. y b. & - Amartya a., b. y c. \\
& - Amartya c. & - Consumidores Argentinos a. y c. \\
& - Consumidores Argentinos c. & - Vida Silvestre a. \\
& - Vida Silvestre a. y b. & - Fortalecer a. y b. \\
& & - Icecor a., b. y c. \\
\hline \multirow{4}{*}{ Nomprando } & Boycotting & Simplifiying \\
& - Consumidores Argentinos c. & - IADS a. y c. \\
& - Vida Silvestre a. y c. & - Amartya a., b. y c. \\
& & - Consumidores Argentinos b. y c. \\
& & - Vida Silvestre a. \\
\hline
\end{tabular}

FUENTE: Elaborado a partir de Valor, 2011: 12.

En términos generales, el buycotting está centrado en productos locales y productos sostenibles que incluyen criterios de compra sociales y ambientales; el smart shopping en comercio justo y agricultura orgánica; el boycotting es de producto (merluza pequeña y agua embotellada) y el simplifying se centra en reciclaje, reutilización, ahorro energético y reducción del nivel de consumo.

- ¿Sobre qué agente actúan las organizaciones en sus proyectos y campañas?

Si nos fijamos en la tabla 9, podemos comprobar la destacable focalización en iniciativas para fomentar el consumo político entre los ciudadanos, ya sea dirigiéndose a ellos de forma individual -por la calle 0 a través de internet- 0 colectiva -en un centro de jubilados o una escuela-. Todas las OSFL actúan sobre este agente, por lo que deducimos que el papel del ciudadano como palanca de cambio es central para las mismas. Además, podemos calificarlas como estrategias de cambio bottom-up, con un enfoque descentralizado y en la base, en la participación de la comunidad (Moyano Estrada, 2005). 


\section{Tabla 9. Agentes sobre los que actúan las OSFL analizadas}

\begin{tabular}{|l|l|l|}
\hline Empresas & Ciudadanos & Administraciones públicas \\
\hline & - IADS a. & \\
- IADS c. & - Amartya a., b. y c. & \\
- Vida Silvestre a. y b. & - Consumidores Argentinos a., b. y c. & - IADS b. \\
- Fortalecer a. y b. & - Vida Silvestre a., b. y c. & - Vida Silvestre a. \\
- Icecor a., b. y c. & - Fortalecer a. & \\
& - Icecor a. y b. & \\
\hline
\end{tabular}

FUENTE: Elaboración propia.

En segundo término se sitúan las empresas, fundamentalmente de pequeño y mediano tamaño, en su rol de productores -en gran parte agropecuarios- así como de consumidores intermedios -de energía o materias primas-. Por último encontramos a las administraciones públicas. Podemos calificar los dos últimos grupos como estrategias de cambio top-down, con un enfoque centralizado (Moyano Estrada, 2005).

\section{6.- Conclusiones}

De acuerdo con los resultados obtenidos tras el estudio de la muestra, varios fueron los hallazgos:

a) Desde el punto de vista de las estrategias de cambio utilizadas por las OSFL, predominan aquellas que no buscan beneficios para las propias organizaciones y que establecen una relación cooperativa. Por consiguiente, observamos una fuerte confluencia de estrategias en uno de los cuatro tipos posibles: promoviendo el cambio.

Las estrategias basadas en el conflicto presentan un peso mucho menor. En principio, este hallazgo es coherente con lo apuntado por Luna y Cecconi (2004): al haber una pequeña presencia OSFL de advocacy, que perciben el poder corporativo como una amenaza y pretenden ejercer un contrapoder, hay pocas estrategias destinadas a forzar y a producir el cambio. 


\section{EL CONSUMO POLÍTICO EN ARGENTINA: ESTRATEGIAS DESARROLLADAS POR ORGANIZACIONES SIN FINES DE LUCRO}

Los dos hallazgos anteriores también pueden explicarse porque Argentina no tiene una gran tradición de OSFL políticas ni de regulación civil. El enfoque predominante históricamente ha sido asistencial, más que transformador. Además, cabe recordar que las relaciones políticas entre OSFL y empresas son muy nuevas en el contexto internacional (Valor y Merino, 2009).

b) Desde el punto de vista de las acciones que puede realizar el consumidor sobre las que inciden las OSFL, observamos una mayor distribución entre los distintos tipos que en el caso de las estrategias de cambio, si bien existe inclinación hacia las formas alternativas: smart shopping y simplifying.

Esta inclinación puede resultar paradójica, ya que implica promover un cambio de sistema, sin embargo hemos visto que son pocas las estrategias que confrontan con las corporaciones convencionales. Podría deberse a la pequeña envergadura y poca antigüedad de la mayoría de OSFL analizadas, características que resultan consistentes con el hecho de que apenas propongan boycotting. Esta práctica de eficacia compleja requiere gran especialización en consumo político por parte del organizador y el éxito puede depender de su alta notoriedad y reconocimiento en la opinión pública, cualidades difíciles de reunir por OSFL pequeñas y de corta trayectoria.

c) En referencia al agente sobre el que actúan las organizaciones, encontramos un predominio de los ciudadanos sobre las empresas y las administraciones públicas. Así pues, son de gran relevancia las estrategias de cambio bottom-up. Este hallazgo es coherente con los anteriores, puesto que implica una menor focalización en los núcleos de poder y explica la mayor orientación a la cooperación. De acuerdo con esta estrategia, el consumo político podría verse como una forma de activismo cotidiano, una creación de poder desde abajo hacia arriba (Micheletti y Follesdal, 2007) que favorece la participación política y por ende, el desarrollo.

d) A partir del caso argentino podemos reflexionar sobre las implicaciones para otros países en desarrollo. Para escenarios en los que el consumo político no tenga una incidencia importante -por el modesto desarrollo de los factores de mercado y/o políticos- y donde predominen las OSFL no transformadoras y asistencialistas, nos encontraremos en mayor medida con estrategias de colaboración para el fomento del consumo político -en detrimento de las conflictivas-, y del tipo bottom-up. Esto significa que las corporaciones no se sentirán lo suficientemente presionadas por las OSFL como para realizar grandes cambios en su conducta -hacia más RSC-, los cuales dependerán de la suma de las iniciativas individuales de los consumidores y de los pequeños emprendedores.

No obstante, hemos de ser prudentes al extrapolar estos resultados a otros territorios, ya que las instituciones políticas condicionan el desempeño de las OSFL, y éstas varían considerablemente entre países (Campbell, 2007). 
Por otra parte, aunque a través del consumo político pueden generarse importantes cambios en el comportamiento de las empresas, para conseguir verdaderas transformaciones en el mercado de forma que éste se alinee con objetivos de desarrollo humano y de sostenibilidad, consideramos necesaria la intervención de los gobiernos.

En este sentido, si bien a mayor presión por parte de las OSFL las empresas serán más responsables, en el largo plazo, los intentos de las OSFL para influenciar el comportamiento corporativo serán más exitosos cuanto más consigan movilizar a otros dos actores: consumidores y gobiernos (Winston, 2002). Por tanto, señalamos como factor clave la formación en RSC para los profesionales (específica) y para la sociedad civil (general), favorecedora del empoderamiento social en derechos humanos que la RSC puede potenciar: de segunda o tercera generación en países con leyes garantistas; de primera generación en países menos desarrollados (Navarro García y García-Marzá, 2009).

Por último, si pensamos en líneas de investigación futuras, sería necesaria mayor información sobre los determinantes macro del movimiento en Argentina, como desarrollo de la demanda, cuotas de mercado de productos responsables o penetración de etiquetas. Asimismo, podría complementarse con estudios enfocados en el consumidor: hábitos de consumo político, dificultades a la hora de ejercerlo o reconocimiento y credibilidad de las etiquetas. Serían óptimos los análisis en tres niveles: provincial, nacional y regional, para poder realizar comparaciones entre territorios argentinos y con países del entorno.

También resultaría interesante estudiar la relación causa-efecto entre desarrollo del consumo político en Argentina y líneas de acción de las OSFL. ¿Las características de las OSFL motivan que el fenómeno no tenga más fuerza o por el contrario son otros actores quienes no presionan suficientemente a su favor? Igualmente, sería importante profundizar en las causas del establecimiento de relaciones de cooperación o conflicto con las corporaciones en el caso argentino, para reflexionar sobre la existencia de tensión y contrapoder suficientes que permitan cambios hacia el desarrollo sostenible. 


\section{Bibliografía}

BANCO MUNDIAL (2015): Argentina / Datos., disponible en http://datos.bancomundial.org/pais/argentina

BARNETT, C., CLARKE, N., CLOKE, P. \& MALPASS, A. (2005a): "Consuming Ethics: Articulating the Subjects and Spaces of Ethical Consumption", Antipode, 37(1), 23-45.

BARNETT, C., CLARKE, N., CLOKE, P. \& MALPASS, A. (2005b): "The political ethics of consumerism", Consumer Policy Review, 15(2), 45-51.

BENDELL, J. (2004): Barricades and Boardrooms: A Contemporary History of the Corporate Accountability Movement, United Nations Research Institute for Social Development (UNRISD), Ginebra.

BENDELL, J. (2007): "The civil regulation of corporation: Towards stakeholder democracy". In: S. Benn \& D. Dunphy (eds.), Corporate governance and sustainability. Challenges for theory and practice, Routledge, Oxon, 206-219.

CAMPBELL, J.L. (2007): "Why would corporations behave in socially responsible ways? An institutional theory of corporate social responsibility", Academy of Management Review, 32(3), 946-967.

CASTRO MONGE, E. (2010): "El estudio de casos como metodología de investigación y su importancia en la dirección y administración de empresas", Revista Nacional de Administración, 1(2), 31-54.

CENTER FOR CIVIL SOCIETY STUDIES (JOHN HOPKINS UNIVERSITY) (2004): Comparative Data Tables (2004), disponible en http://ccss.jhu.edu/wpcontent/uploads/downloads/2013/02/Comparative-data-Tables_2004_FORMATTED_2.2013.pdf

CHERRIER, H. (2007): "Ethical consumption practices: Co-production of self-expression and social recognition", Journal of Consumer Behaviour, 6(5), 321-335.

CORBETTA, P. (2007): Metodología y técnicas de investigación social, McGraw-Hill, Madrid.

COSCIONE, M. (2008): "Las relaciones de comercio justo entre Europa y América Latina. ¿Una posible alianza estratégica para el desarrollo?", Circunstancia, 17(6), 1-12.

DOY, J.P. \& GUAY, T.R. (2006): "Corporate Social Responsibility, Public Policy, and NGO Activism in Europe and the United States: An Institutional-Stakeholder Perspective", Journal of Management Studies, 43(1), 47-73.

ECHEGARAY, F. \& SARSFIELD, R. (2010): "Votando con el "changuito": La politización del consumo en América Latina", Revista Latinoamericana de Opinión Pública, 1(0), 1-26. 
EISENHARDT, K.M. (1989): "Building theories from case study research", The Academy of Management Review, 14(4), 523-550.

ESE (ENCUESTA SOCIAL EUROPEA) (2015): Data and Documentation, disponible en http://www.europeansocialsurvey.org/data/

FUNDACION FORTALECER (2012): Evolución mundial del Sistema de Comercio Justo en el 2011. Situación Argentina y aportes del Programa de Comercio Justo implementado por Fundación Fortalecer-Federación Agraria Argentina, disponible en http://www.fortalecer.com.ar/wp-content/uploads/2012/02/InformeFF2011_EvoluciondelSistemaFairTrade.pdf

GRZYBOWSKI, C. (2001): "Las organizaciones no gubernamentales y la comunicación de masas: posibilidades de movilización", Comunicar, 16, 25-32.

KLINTMAN, M. (2006): "Ambiguous framings of political consumerism: means or end, product or process orientation?", International Journal of Consumer Studies, 30(5), 427-438.

KOZINETS, R.V. \& HANDELMAN, J.M. (2004): "Adversaries of Consumption: Consumer Movements, Activism, and Ideology", Journal of Consumer Research, 31(3), 691-704.

LOW, W. \& DAVENPORT, E. (2007): "To boldly go...exploring ethical spaces to re-politicise ethical consumption and fair trade", Journal of Consumer Behaviour, 6(5), 336-348.

LUNA, E. \& CECCONI, E. (Coords.) (2004): Índice de Desarrollo Sociedad Civil de Argentina. Total País, Programa de las Naciones Unidas para el Desarrollo, Banco Interamericano de Desarrollo y Grupo de Análisis y Desarrollo Institucional y Social.

MATEOS, M. \& GHEZÁN, G. (2010): "El proceso de construcción social de normas de calidad en alimentos orgánicos y la inclusión de pequeños productores. El caso de Argentina". En: Innovation and Sustantaible Development in Agriculture and Food, Montpellier.

MICHELETTI, M. \& FOLLESDAL A. (2007): "Shopping for human rights. An introduction to the special issue", Journal of Consumer Policy, 30(3), 167-75.

MICHELETTI, M. (2002): "Consumer choice as political participation", Statsvetenskaplig Tidskrift, 105(3), 218-234.

MOVIMIENTO ARGENTINO POR LA PRODUCCIÓN ORGÁNICA (2013): ¿Qué es la producción orgánica?, disponible en http://www.mapo.org.ar/que-es-la-produccion-organica

MOYANO ESTRADA, E. (2005): Capital social y desarrollo en zonas rurales, Instituto de Estudios Sociales de Andalucía (IESA-CSIC).

NAVARRO GARCÍA, M. \& GARCÍA-MARZÁ, D. (2009): "La RSC en el marco de la cooperación al desarrollo y la internacionalización de la empresa española en países de renta media y rehabilitación postbélica", Revista Española del Tercer Sector, 11, 115-143.

NOVO VÁZQUEZ, A. (2014): "“'Consumocracia”. El consumo político como forma de participación de la ciudadanía", Política y Sociedad, 51(1), 121-146. 


\section{EL CONSUMO POLÍTICO EN ARGENTINA: ESTRATEGIAS DESARROLLADAS POR ORGANIZACIONES SIN FINES DE LUCRO}

PALADINO, M. \& MOHAN, A. (2002): "Tendencias de la Responsabilidad Social Empresaria en Argentina", IAE - Escuela de Dirección y Negocios de la Universidad Austral, Pilar.

PAPAOIKONOMOU, E. (2012): "Sustainable lifestyles in an urban context: towards a holistic understanding of ethical consumer behaviours. Empirical evidence from Catalonia, Spain", International Journal of Consumer Studies, 37(2), 181-188.

PINZÓN CAMARGO, M.A. (2005): "El papel de las ONG y sus alcances en la Responsabilidad Social Empresarial", Revista Opera, 5(5), 75-96.

PNUD (PROGRAMA DE LAS NACIONES UNIDAS PARA EL DESARROLLO) (2013): Informe sobre Desarrollo Humano 2013. El ascenso del Sur: Progreso humano en un mundo diverso, PNUD, Nueva York.

PNUD (PROGRAMA DE LAS NACIONES UNIDAS PARA EL DESARROLLO) (2014): Informe sobre Desarrollo Humano 2014. Sostener el Progreso Humano: Reducir vulnerabilidades y construir resiliencia, PNUD, Nueva York.

ROITTER, M.M. \& GONZALEZ BOMBAL, I. (comps.) (2000): Estudios sobre el Sector Sin Fines de Lucro en Argentina, The Johns Hopkins University- Centro de Estudios de Estado y Sociedad.

SAGUIER, M.L. (2005): Las transformaciones recientes de la sociedad civil en la Argentina: economía social y organizaciones de trabajadores desocupados, disponible en http://www.equitativo.com.ar/icecor/documentos/doc_ecosocialyorgtrabdeso_saguier.htm\#_ftn1

SENASA (SERVICIO NACIONAL DE SANIDAD Y CALIDAD AGROALIMENTARIA) (2014): Situación de la Producción Orgánica en la Argentina durante el año 2013, disponible en http://www.mapo.org.ar/wp-content/uploads/2014/05/informe-senasa-2013.pdf

STOLLE, D. \& MICHELETTI, M. (2005): What motivates Political Consumers?, disponible en http://www.politik-konsum.de/en/pdf/fnsb_stolle_micheletti.pdf

THOGERSEN, J. (2010): "Country Differences in Sustainable Consumption: The Case of Organic Food", Journal of Macromarketing, 30(2), 171-185.

VALOR, C. \& MERINO DE DIEGO, A. (2009): "Relationship of business and NGOs: an empirical analysis of strategies and mediators of their private relationship", Business Ethics: A European Review, 18(2), 110-126.

VALOR, C. (2011): Ciudadanos de mercado y consumidores políticos, Universidad Nacional de Educación a Distancia (UNED).

VARGAS, J.G. (2003): "Teoría de la acción colectiva: sociedad civil y movimientos sociales en las nuevas formas de gobernabilidad en Latinoamérica", Nómadas, 7.

WINSTON, M. (2002): "NGO Strategies for Promoting Corporate Social Responsibility", Ethics \& International Affairs, 16(1), 71-87. 
